\title{
Anomalously High Conductivity of Deformed Metals at the Positive Temperatures
}

\author{
Gennady A. Markov ${ }^{1}$, Vladimir N. Malyshev ${ }^{2}$ \\ ${ }^{1}$ Technologies of Markov, Ltd., Novosibirsk, Russia; ${ }^{2}$ Mechanical Engineering Department, Gubkin Russian State University of Oil \\ and Gas, Moscow, Russia. \\ Email: markova-nsk@mail.ru,vmal@inbox.ru
}

Received January $19^{\text {th }}, 2012$; revised February $14^{\text {th }}, 2012$; accepted February $26^{\text {th }}, 2012$

\begin{abstract}
The description of experimentally observed phenomenon of abnormally high electrical conductivity- "superconductivity" (SC) at the room and higher temperatures is represented. The effect was observed in metallic monospirals of small radius curvature with high density and regular distribution of dislocations. Transition into state of SC has been observed experimentally in the range from $-50^{\circ} \mathrm{C}$ up to $3000^{\circ} \mathrm{C}$ at the density of transmitting current up to $2 \times 10^{9} \mathrm{~A} / \mathrm{cm}^{2}$. The experimental data confirming the watched phenomenon are represented. The explanations of this phenomenon are being proposed in the framework of the dislocation model.
\end{abstract}

Keywords: Metallic Monospirals; Dislocation Density; The Anomalously High Conductivity in Metals-Superconductivity; Room and Other Positive Temperatures; The Velocity of Current Density Growth

\section{Introduction}

When you create a production technology of tungsten spirals with very small radius of curvature, the study of mechanical and physical properties of these helixes, yet in the 80 s of the 20th century was experimentally discovered the effect of abnormally high increase their electrical conductivity at the room temperatures $[1,2]$. This effect resembled the phenomenon of superconductivity, discovered in 1911 by Dutch physicist H. Kamerlingh Onnes in metals at temperatures close to absolute zero [3].

Under the superconductivity (SC) in metals since that time are understood the sharp decline of resistivity of the conductor to almost zero and the "unimpeded" flow of electric current through it. This gives the possibility in future to transmit the electric power over long distances without loss.

Until now scientists are hoping to get the matter in superconducting state at the room temperature (300 - 400 $\mathrm{K})$. When, where, and in what of materials can be implemented such kind of superconductivity?

According to Nobel Prize Laureate Vitaly Ginzburg [4], "...we have a natural boundary-2011 year i.e. the centenary of the discovery of superconductivity".

\section{Method of Producing the Superconducting State in Metallic Monospiral at the Positive Temperature}

To obtain the desired effect obviously has to be activated mechanism that is different from the BCS mechanism [3]. To do this in the metallic conductor has to be created special channels-waveguides for unimpeded movement of charges through the conductor. We argue that such channels can be formed in the metal in which are created, for example, planes of dislocations evenly spaced along the length of the conductor, due to its plastic deformation [5-7].

For transition of metallic conductor into state of abnormally high conductivity should be performed four basic conditions:

1) The necessary dislocation density (from $10^{8} \mathrm{~cm}^{-2}$ and above) in the conductor should be created;

2) Their regular distribution along the length of the conductor should be executed;

3) High velocity of current density growth (from $10^{2}$ $\mathrm{A} / \mathrm{cm}^{2} \cdot \mathrm{s}$ and above) should be ensured;

4) The necessary temperature for transition into superconducting state should be reached.

Necessary dislocation density can be achieved by various methods of deformation: by bending, rolling, squeezing and stretching. However, the listed above methods of metal deformation can't to provide regular distribution of dislocations along whole length of the conductor. To ensure both the high density of dislocations and simultaneously their regular distribution along the length of the conductor it is necessary to carry out either winding the wire into monospiral or twisting of two wires into spiral. In this case, the internal diameter 
of each monospiral tends to zero, and the outer diameter of helix tends to two diameter of the wire, which results to maximum packing density, i.e. the larger the angle of the wire torsion direction to the longitudinal axis, the higher is the density of dislocations.

\section{Experiment: Research Methods and Equipment, Materials, Technology for Creating of Samples}

The conductor was made from tungsten wire with diameter $0.05 \mathrm{~mm}$ by means of its torsion into monospiral. Conductor was placed into a chamber with an inert gas and connected to current feeds. Current-voltage characteristics were fixed and examined on a computer. The current increasing in time was given on the conductor in the mode of stabilization. Any given magnitude of the current was remained constant regardless of changes in the resistance of the conductor at any time. Change the current in time was given by the regulator of current stabilization. Necessary heating of monospiral up to temperature of transition it into superconducting state was carried out by way of passing current.

The increase of current density was being conducted up to a stop of growth of voltage in monospiral. Stopping of voltage increase is the beginning of transition of the conductor into state with anomalously high conductivity - state of SC. The further insignificant increase of current density led to voltage reduction up to zero that testified about sharp decrease of resistance of monospiral and its transition into state with abnormally high conductivity.

To confirm this effect the samples not only from tungsten, but also from copper, tantalum, aluminum and other metals were taken and subjected to plastic deformation by torsion up to the dislocation density not lower than $10^{8}-10^{12} \mathrm{~cm}^{-2}$.

The preparation of samples conductors was carried out by winding of wire with diameter $d$ into monohelix with step $h$ on cord thread with diameter $d_{0}$. The degree of plastic deformation was determined as:

$$
\varepsilon= \pm d / D \cos \alpha
$$

where $D=d+d_{0}$ : the diameter of the helix, $\alpha$ : the angle of winding spiral turns. The + sign denotes the outer side of the helix.

Pitch of helix was given based on:

$$
h=\pi D \operatorname{tg} \alpha
$$

From this it follows that $\varepsilon \rightarrow 1$, when $d \square d_{0}$, as $h \rightarrow$ $d$. To obtain the desired degree of plastic deformation $h$ and $d_{0}$ can be changed.

Spiral wires were fixed in a vacuum chamber on isolated brackets. The ends were connected to current supply. The air was evacuated from the chamber and filled with an inert gas (helium, argon or nitrogen) under pressure changing from low to atmospheric pressure.

Conductivity measurement was carried out on laboratory installation (Figure 1) which includes the capacitors 1 (100 - 800 microfarads, charged up to $300-800 \mathrm{~V})$, the air gap (discharger) or the high-speed thyristor 2 , the block of high voltage 3 , the investigated conductor 4 , the measuring shunt 5 , the oscilloscope 6 , the computer 7 for fixation the high-speed current-voltage characteristics.

For comparison of the conductivity two conductors were taken. One of them was executed in the form of cylindrical monospiral winded from tungsten wire with diameter of $0.05 \mathrm{~mm}$ and length of $100 \mathrm{~mm}$, while the other was an ordinary from copper wire with diameter 4 $\mathrm{mm}$ and $50 \mathrm{~mm}$ in length. These conductors were placed alternately in the installation and connected to currentsupplying feeds.

The capacitor 1 was charged by current source 3 up to fixed voltage, for example, up to $600 \mathrm{~V}$. Then, the current source was turned off and the air discharger 2 switched on. The current was transmitted onto monospiral 4 , which was heated up in a pulsed mode in time $-1.5 \times$ $10^{-3} \mathrm{~s}$ and passed into the superconducting state.

It is necessary to pay attention that the current in the circuit "capacitor-air discharger-monospiral" was increased not at once from the moment of discharger switching on, but only after warming up of monospiral up to temperature of its transition into superconducting state that was occurred through $1.5 \mathrm{~ms}$ from the moment of discharger switching on (see Figure 2).

Further, instead monospiral, the circuit was closed by the short thick copper conductor with diameter of $4 \mathrm{~mm}$. At the capacitor discharge of the same capacitance as in the first case and with the same charging voltage, the current in the circuit "capacitor-air discharger-copper conductor," grew immediately after 0.1 milliseconds up to the same value in $600 \mathrm{~A}$, as when was in circuit the monospiral. The ultimate magnitude of the current was limited by total inductance of the power supply.

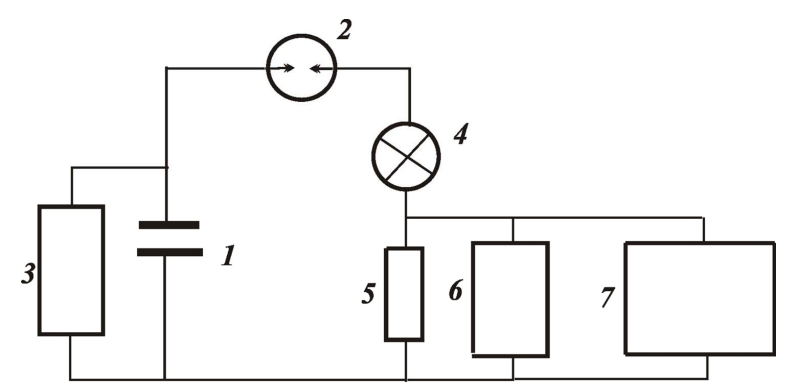

Figure 1. Schema of laboratory installation: 1) Capacitor; 2) Air discharger or high-speed thyristor; 3) Block of high voltage; 4) Tested conductor; 5) Measuring shunt; 6) Oscillograph; 7) High-speed xy-plotter (computer). 
Besides, the experiments also were carried out in according to the following technique. On the conductor was transmitted the direct current increasing in time, but in the mode of stabilization. The magnitude of the current remained constant regardless of the load at any given time, and the difference of potential across the load depended only from the resistance of the conductor. Regulator of currents stabilization allows changing the value of current in the linear dependence. Velocity of current density growth was set in a range from 100 up to $2 \times 10^{5} \mathrm{~A} / \mathrm{cm}^{2} \cdot \mathrm{s}$. This increase of the current density in the conductor were conducted up to the total voltage drop in it, on schedule-controlled volt-ampere characteristics (VAC) which testified about the transition of conductor into superconducting state. In reverse mode, at the reducing the current density in the stabilization mode the loop of hysteresis was appeared. This experiment was carried out in automatic mode of input and output into the state of SC duration to 100 hours of continuous operation.

\section{The Results of Research}

For the pulsed mode the characteristics of current variations with time for monospiral from tungsten wire with diameter of $0.05 \mathrm{~mm}$ and conductor from copper wire with diameter of $4 \mathrm{~mm}$ on Figure 2 are shown.

The typical dependence of voltage changes of deformed tungsten wire conductor from current in mode of stabilization on Figure 3 is shown.

The plastic deformation of tungsten wire was carried out at the room temperature. The curve $A B C D E F$ (see Figure 3) is composed from three parts with different electrical resistivity. The deviation of $U$ from its constant value on the segment $A B$ is connected with the heating of wire and increasing its resistance. The dashed curve $B C$ corresponds to the dependence of electrical resistance from temperature for not deformed conductor or for annealed after its deformation at $T>0.5 T_{m}$ within the time more than $10^{3} \mathrm{~s}$. Here $T_{m}$ is melting temperature.

At the increasing of current density for deformed conductor on the segment $A B$ at the average velocity greater than $2 \times 10^{5} \mathrm{~A} \cdot \mathrm{cm}^{-2} \cdot \mathrm{s}^{-1}$ the voltage $U$ deviates from curve $A C$ in the point $B$. The characteristic value of the external electric field $E_{c}$ is the magnitude about $10^{2} \mathrm{~V} / \mathrm{m}$. On the part of the curve $B D$ on Figure 3 is shown that the voltage on the conductor decreases. The characteristic oscillations of dependence $U(I)$ are observed also.

Length of the $B D$ depends on the type of the metal and its degree of deformation. The curve $B F$ is constructed for the velocity of current density growth equal to $5 \times$ $10^{5} \mathrm{~A} \cdot \mathrm{cm}^{-2} \cdot \mathrm{s}^{-1}$. On the segment $D E$ the reduction of resistance is even more pronounced than on the previous one. At the point $F$ the resistivity $\rho$ of deformed wire is ap- proximately in 22 times less than at the point $A$. With further increase of current density, the resistivity $\rho$ tends to zero. This corresponds to decrease of electrical resistance in $30-100$ times. With increasing of current density the good electrical hysteresis is manifested.

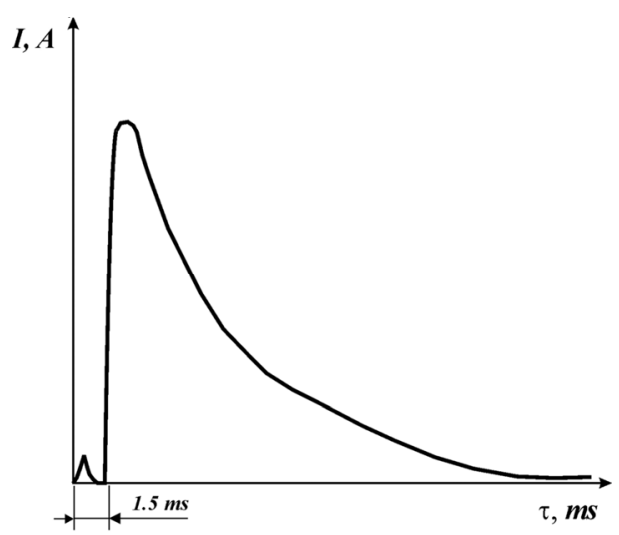

(a)

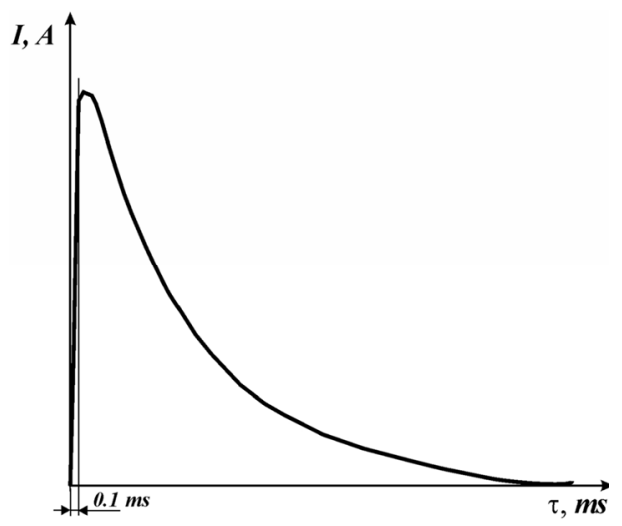

(b)

Figure 2. Characteristic dependences of current changes in time in pulse mode for: (a) Tungsten monospiral $0.05 \mathrm{~mm}$; (b) Copper wire $4 \mathrm{~mm}$.

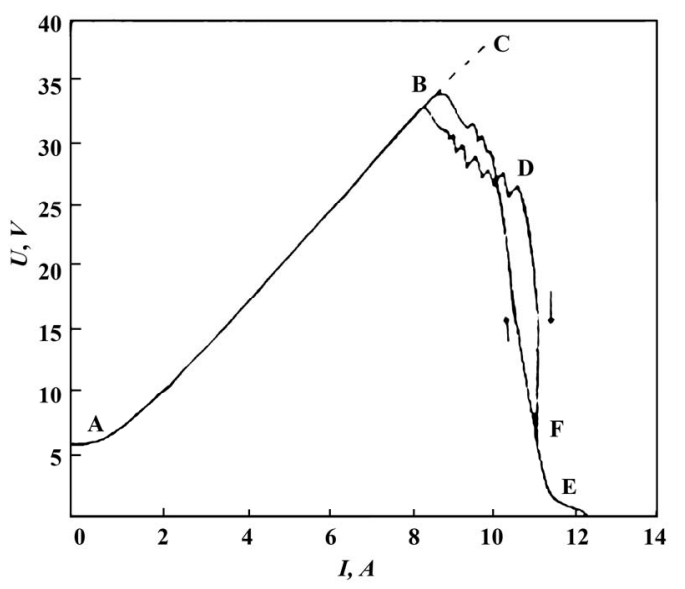

Figure 3. Voltage-current characteristic of tungsten monospiral conductor. 
The dislocation density in the cylindrical monospirals obtained by plastic deformation, calculated using the formula:

$$
n=\frac{k}{b(r+h) \cos \beta}
$$

where $n$ : the dislocation density; $b$ : Burger's vector; $r$ : mean radius of monospiral; $h$ : the diameter of the conductor; $\beta$ : angle between the line of the middle circle of monospiral and the plane slip of dislocation; $k$ : the relation of the conductor diameter to the inside diameter of monospiral.

Verification of the dislocation density was performed with an electron microscope, and then was determined the transition temperature of the conductor into superconducting state. The critical transition temperature into $\mathrm{SC}$ state and the conditions for this transition was calculated using the formula:

$$
t_{c r}=\frac{t_{m}}{\lg \left(\frac{n}{n_{c r}} \times \frac{j_{c}}{j_{c r}}\right)}
$$

where: $t_{c r}$ : critical transition temperature into $\mathrm{SC} ; t_{m}$ : melting temperature of the conductor; $n_{c r}$ : the critical density of dislocations for transition into $\mathrm{SC} ; n$ : the dislocations density in the conductor; $j_{c r}$ : the critical current density for transition into $\mathrm{SC} ; j_{c}$ : the velocity of current density growth in the conductor.

Formulas (3) and (4) allow to calculate all the parameters necessary for the converting the metal wire into superconducting state. For example, we need a transition temperature into state of SC at the $1250^{\circ} \mathrm{C}$. According to formula (3) we calculate the necessary dislocation density for the tungsten wire and define that it should be 5.7 $\times 10^{10} \mathrm{~cm}^{-2}$. Calculated diameters of cylindrical monospirals for tungsten wires of two diameters 0.025 and $0.050 \mathrm{~mm}$ should be accordingly 0.081 and $0.130 \mathrm{~mm}$.

Note, that by plastic deformation can be achieved the dislocation density not greater than $1 \times 10^{14} \mathrm{~cm}^{-2}$. At this density of dislocations the transition temperature for the conductor into superconducting state is quite high. The dependences of transition temperature into superconducting state from the dislocation density and the velocity of current density growth in three-dimensional space on Figure 4 are shown.

When the dislocation density $1 \times 10^{8} \mathrm{~cm}^{-2}$ and the velocity of current density growth $2 \times 10^{5} \mathrm{~A} \cdot \mathrm{cm}^{-2} \cdot \mathrm{s}^{-1}$ the transition temperature into superconducting state for tungsten is $3410^{\circ} \mathrm{C}$. The magnitude of the dislocation density $5 \times 10^{15} \mathrm{~cm}^{-2}$ is the experimental result achieved by heat treatment of metallic conductors. With this dislocation density the transition of the conductor into superconducting state occurs at the room temperature $\left(20^{\circ} \mathrm{C}\right)$.
The magnitude of the dislocation density $1 \times 10^{15} \mathrm{~cm}^{-2}$ mechanically (by plastic deformation) can't be reached because of saturation, however, thanks to the heat treatment in experiments at the velocity of current density growth $1 \times 10^{4} \mathrm{~A} \cdot \mathrm{cm}^{-2} \cdot \mathrm{s}^{-1}$ we are able to get repeatedly the transition into superconducting state at $10^{\circ} \mathrm{C}-20^{\circ} \mathrm{C}$. Stable results correspond to the three-dimensional diagram (Figure 4) and are the magnitudes specified on Table 1.

Thus, by means the heat treatment of conductor can be increased the dislocation density to values of about $1 \times$ $10^{15}-2 \times 10^{15} \mathrm{~cm}^{-2}$, which reduces the temperature of transition into superconducting state.

Similar investigations were conducted with wires from other metals such as copper, nickel, aluminum, tantalum, molybdenum. They are showed that by heat treatment is possible to achieve such density of dislocations which decreases the threshold for the transition of metal into superconducting state (see Table 2).

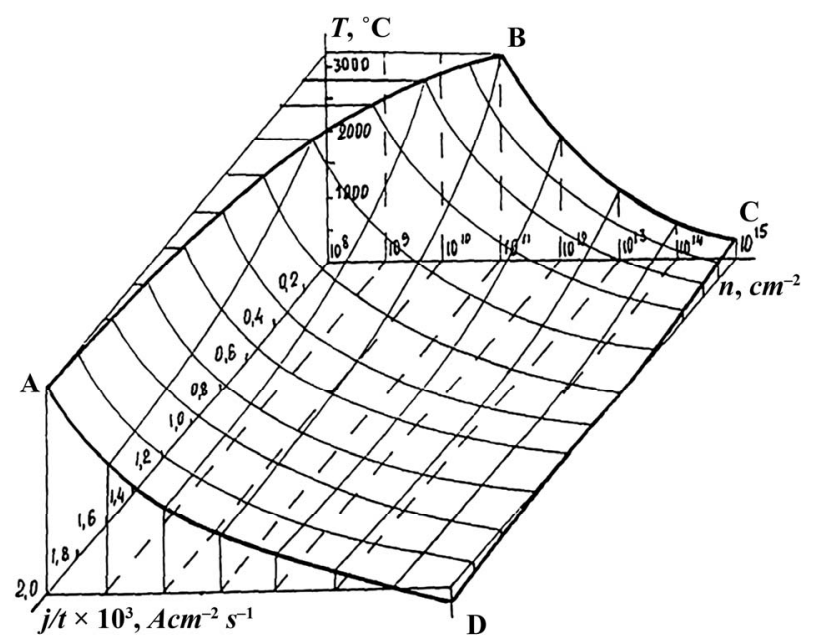

Figure 4. Three-dimensional diagram of dependence of dislocations density, velocity of current density growth and transition temperature of monospiral conductor into superconducting state.

Table 1. Dependence of dislocations density, velocity of current density growth and transition temperature into superconducting state for tungsten.

\begin{tabular}{ccc}
\hline $\begin{array}{c}\text { Dislocations } \\
\text { density } \\
\mathrm{cm}^{-2}\end{array}$ & $\begin{array}{c}\text { Velocity of current } \\
\text { density growth } \\
\mathrm{A} \cdot \mathrm{cm}^{-2} \cdot \mathrm{s}^{-1}\end{array}$ & $\begin{array}{c}\text { Transition temperature into } \\
\text { superconducting state } \\
{ }^{\circ} \mathrm{C}\end{array}$ \\
\hline $1 \times 10^{14}$ & $1 \times 10^{4}$ & 500 \\
$1 \times 10^{14}$ & $2 \times 10^{5}$ & 20 \\
$1 \times 10^{15}$ & $1 \times 10^{4}$ & 100 \\
$1 \times 10^{15}$ & $2 \times 10^{5}$ & -50 \\
\hline
\end{tabular}


Table 2. Dependence of dislocations density, velocity of current density growth and transition temperature into superconducting state for some metals.

\begin{tabular}{lccccc}
\hline Materials & Cu & Ni & Al & Ta & Mo \\
\hline $\begin{array}{l}\text { Dislocations } \\
\text { density, } \mathrm{cm}^{-2}\end{array}$ & $1 \times 10^{11}$ & $9 \times 10^{10}$ & $1 \times 10^{13}$ & $3 \times 10^{11}$ & $9 \times 10^{10}$ \\
$\begin{array}{l}\text { Velocity of current } \\
\text { density growth, } \\
\mathrm{A} \cdot \mathrm{cm}^{-2} \cdot \mathrm{s}^{-1}\end{array}$ & $1 \times 10^{5}$ & $2 \times 10^{5}$ & $1 \times 10^{5}$ & $6 \times 10^{4}$ & $1 \times 10^{5}$ \\
$\begin{array}{l}\text { Transition temperature } \\
\text { into SC state, }{ }^{\circ} \mathrm{C}\end{array}$ & 900 & 1300 & 600 & 2000 & 2000 \\
\hline
\end{tabular}

\section{Discussion}

An explanation of described phenomenon in the framework of classical electronic theory, as well as within the well-known BCS-theory of superconductivity is not possible, therefore we can suppose about some another type of superconductivity in metals. The authors propose that this phenomenon can be explained from the standpoint of dislocation theory.

Firstly about it says that the conductor prepared by this method has the form of infinite monospiral and it means that it has the form of infinite linear inductance in which at the certain parameters the own magnetic field is equal the zero, and, hence, absent of limit on infinite growth of current density in the conductor. It is known that both for usual low-temperature superconductors and for HTSCsuperconductors the magnetic field is one of the important parameters limiting their applying by density of current. The second positive moment of such type of superconductivity for the conductor having the form of monospiral is its high flexibility without destruction of superconducting structure of metal, in difference from HTSC-2 cable having a heightened fragility of current-carrying ceramics. In this case are absent the limits practically for diameter of wire, let alone that is eliminated the necessity for cryogenic technology.

It was been established experimentally that at the approach of dislocations density up to $1 \times 10^{15} \mathrm{~cm}^{-2}$ and at the maximum velocity of current density growth $\left(2 \times 10^{5}\right.$ $\left.\mathrm{A} / \mathrm{cm}^{2} \cdot \mathrm{s}\right)$ it is possible to provide the transition into superconducting state at $-50^{\circ} \mathrm{C}(223 \mathrm{~K})$. Increasing of dislocation density from $1 \times 10^{8}$ to $1 \times 10^{15} \mathrm{~cm}^{-2}$ was obtained by heat treatment of wire, deformed preliminarily mechanically. The samples were specially deformed by their twisting into monospiral with an inner radius of curvature, tending up to zero. Thus, the geometrical redistribution of atoms is occurred on the inner (small curvature) and outer (large curvature) surfaces, which form a kind of dislocations walls or so-called dislocations planes. These walls are formed by infinite parallel edge dislocations with equal Burger's vectors that are described, for example, by M. A. Shtremel [8] and Zh. Friedel [9].

Heat treatment (the polygonization) as a result of slip and climb of dislocations is the process of dividing the grains into fragments. Polygons and groups of dislocations of identical characters are grouped into the walls (Figure 5).

The formed planar dislocation walls are inactive and very stable. With further heating, they are retained almost to the melting point of metals. After the formation of subgrain structure the recrystallization process is not occurred. At the passing an electric current through a metal conductor twisted into monospiral with a large radius of curvature and at the velocity of current density growth more than $100 \mathrm{~A} \cdot \mathrm{cm}^{-2} \cdot \mathrm{s}^{-1}$, the passage of electrons occurs between the formed walls of dislocationswaveguides.

When heated, the deformed twisting conductor over $3000^{\circ} \mathrm{C}$, in it formed the single-crystal blocks along the conductor length of 0.5 to 2 diameters of the wires with high dislocation density, which can be observed in the microscope. The wire thus becomes not round and represents packing of blocks located along a wire. These blocks have the various forms depending on degree of curvature and owing to high density of dislocations. A lot of dislocation planes forming the waveguides for current flow are generated by twisting the wire in the form of cylindrical monospiral with certain parameters.

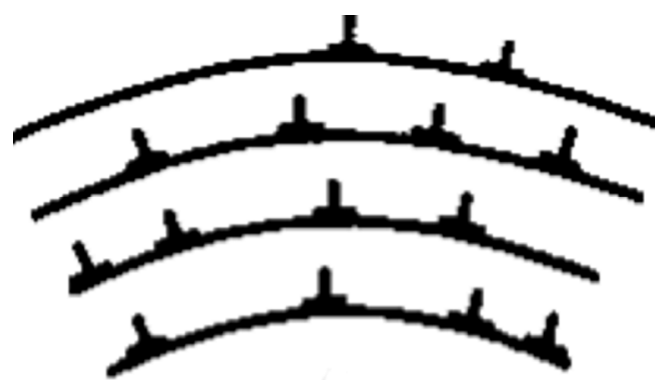

(a)

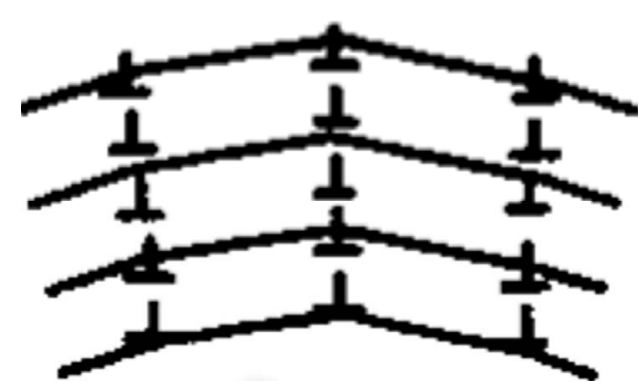

(b)

Figure 5. Schema of polygonization: (a) Chaotic orientation of dislocations in deformed metal; (b) The dislocation walls after process polygonization. 
The principle of current flow in the channels formed with such dislocation planes in the metals deformed in this manner is similar to a passage of light in the fiberoptical cable used for transmission over long distances without loss of luminous flux.

The flow of current during the transition it from "superconductor" to the copper wire is accompanied by the melting area of the copper wire of length $10-30 \mathrm{~cm}$. And this despite the fact that the density of transmitted current is in ten times lower than the current density, which can warm up this conductor to the temperature of $50^{\circ} \mathrm{C}$ $150^{\circ} \mathrm{C}$. Attention is drawn to the fact that itself this metallic superconductor has the ambient temperature, i.e. about $25^{\circ} \mathrm{C}-30^{\circ} \mathrm{C}$. The facts of transition an electronic current from metallic monospiral superconductor to usual copper conductor having flexures are interesting also. The melting of copper wire is occurred on the larger radius of curvature, but the smaller radius remains unchanged, which also confirms the non-classical version of the electrons motion.

If for metallic superconductor is applied the direct current, it is heated the copper wire feeding with a positive potential, while the lead wire with negative potential is not warmed up.

Note, that if the helix is heated by way of electric current in an inert atmosphere up to temperature $2500^{\circ} \mathrm{C}$, then the monospiral pass into the state of superconductivity, and the temperature of the helix is reduced up to room temperature and it will be in the SC state so long as in it the current is flowing.

To reduce the time required to reach the transition of the conductor into SC state at the room temperature is necessary to use an external heat source up to $3000^{\circ} \mathrm{C}$. Several thousand samples was verified experimentally on the stability of conductors transition into SC state as a functions of heating temperature, velocity of current density growth and dislocation density.

The measuring of transition temperature into superconducting state on voltage-current characteristics was conducted for improvement of thermal tempering methods with the purpose of magnification of dislocations density due to polygonization process and for control of dislocations density. Moreover, for each specific sample the transition temperature persists for the multiple (up to 10,000 cycles) transition of the conductor into the state of SC and back with an accuracy of $0.01 \%$.

Methods of heat treatment may be different: the heating by electric current, ion current, plasma discharges, laser, etc. It must ensure that the main objective is the achievement of the dislocation density required for the transition of the conductor into superconducting state. In metallic superconductors of this type were obtained experimentally the current densities up to $1-2$ billion $\mathrm{A} \cdot \mathrm{cm}^{-2}$, which exceeds in 20,000 times the current density attainable for low-temperature metallic superconductors.

\section{Conclusions}

The model for achieve the SC state in metals at positive temperatures considered in the paper based on the creation in the metallic conductor channels for unobstructed passage of current, whose formation in the form of evenly spaced along the length of dislocation planes is provided by plastic deformation of the torsion and subsequent heat treatment.

The proposed method of converting the metallic conductor into superconducting state at the positive temperatures and particularly at temperatures close to the room temperature opens up principally new opportunities and perspectives of using these materials in various fields of industrial production and energy.

\section{REFERENCES}

[1] G. A. Markov, N. V. Melnikova and Y. A. Khon, "The Anomalously High Conductivity of Deformed Metals," Letters to JTPh, Vol. 22, No. 16, 2002, 1996. pp. 65-72.

[2] Y. A. Khon, G. A. Markov and N. V. Melnikova, "Anomaly High Electrical Conductivity of Deformed Metals for High Electric Current Density," Proceedings of International Conference of Role of Mechanics for Development of Science and Technology, Beijing, 13-16 June 2000, pp. 639-640.

[3] V. Buckel, "Superconductivity," Mir, Moscow, 1975, p. 364.

[4] V. L. Ginzburg and D. A. Kirzhnic, "Problem of HighTemperature Superconductivity," Nauka, Moscow, 1977, p. 400.

[5] G. A. Markov, "Method of Converting Metal Conductor into Superconducting State," Patent No. 2233349 RU, 2002.

[6] G. A. Markov, "Method of Creation Abnormally Conductivity," Patent No. 1826744 RU, 2009.

[7] G. A. Markov, "Method of Creation Abnormally Conductivity," Patent No. 2061084 RU, 2010.

[8] M. A. Shtremel, "Toughness of Alloys. Part 1. Defects of the Grating. The Manual for High Schools," Metallurgy, Moscow, 1982, p. 280.

[9] Zh. Friedel, "Dislocations," Mir, Moscow, 1967. 